\title{
POLITICAS DE JUVENTUD: ENTRE LA FRAGILIDAD Y EL DESCONCIERTO Algunas pistas para construir rutas desde lo local
}

\author{
ANDREA IGLESIS LARROQUETTE*
}

\section{PRETEXTOS Y CONTEXTOS}

Finalizado el milenio, Latinoamérica completa ha tenido experiencias de políticas de juventud; sin embargo, éstas no han contribuido a la solución del principal problema que enfrentan las juventudes en el continente: el de la exclusión social.

En este contexto de ineficacia, el principal problema parece estar asociado al tipo de respuestas simplistas que la política social se ha estado planteando frente al problema, y no únicamente a la falta de recursos y validez política como comúnmente se argumenta (Rodríguez, 2000).

Para nuestro país, dicha consideración pone de manifiesto un elemento más a la hora de tratar de comprender la situación actual de la política de juventud.

Iniciado el tercer milenio en Chile, no existe claridad en torno a quiénes deberían ser las y los sujetos de la política de juventud, cuál es el propósito de la política ni cómo desarrollar o implementar dicha política para alcanzar su propósito.

Psicóloga Egresada de la Universidad de Valparaíso. Encargada del Área de Desarrollo Local y Proyectos, del Departamento de Jóvenes de la I. Municipalidad de Concepción. E-mail: aila@entelchile.net. 
Así vemos que en la actualidad la construcción de sujeto joven oscila entre dos ejes interpretativos, apreciándose a la juventud o como amenaza para la convivencia social, aplicándose entonces medidas restrictivas y de prevención, o como recurso estratégico del futuro de la sociedad, aplicando ahí medidas de promoción y participación (Steigler, 2000).

Esta construcción de problema en torno a la juventud, sin duda determina y orienta la generación de acciones y planes diversos, generándose una ambigüedad que resta orientación y coherencia a la acción pública.

Estas tensiones implican diversas aproximaciones a lo juvenil (dependiendo de las direcciones en que se resuelvan) y su identificación permite interpretar ambigüedades que limitan la coherencia y claridad de una política hacia los jóvenes.

Si a eso se le suma la falta de claridad político institucional y la consecuente des-agregación de actores vinculados a la administración de la oferta social en juventud, es claro que en nuestro país no existe una política de juventud, fundamentalmente porque no existe ni ha existido una visión de conjunto capaz de «concebir la intervención en juventud como una política social juvenil, que supere la fase agregativa y pase a una visión constructiva de políticas en juventud» (Silva, 2000).

De una u otra forma, la política de juventud que hemos sido capaces de desarrollar sobrevive entre la fragilidad (producto de la inconsistencia político-social) y el desconcierto (de las y los actores involucrad@s).

Frente a esta situación, uno de los desafíos más urgente de la política de juventud tiene que ver con la capacidad de re-pensar(se), a través de un proceso de acumulación de experiencias-aprendizajes, que permitan imprimir cambios cualitativos en la gestión de la política pública de juventud.

Así entonces y aun cuando la implementación de la política de juventud en nuestro país ha sido constantemente sometida a análisis que pueden dar cuenta de un período de crisis más o menos aguda durante el último quinquenio, quizás el principal nudo se relaciona con la incapacidad de implementar adecuadamente al menos «un» modelo de política de juventud.

Es posible observar entonces que el problema de fondo no resulta ser la pertinencia del modelo «declarado», vale decir, el problema no se relaciona con la existencia de una instancia central coordina- 
dora especializada en la generación de estudios y evaluaciones que constituyan un apoyo a la gestión de otras instituciones que desarrollan la función de vinculación con las juventudes, sino que el problema es la incapacidad de este organismo central (INJ) para coordinar y orientar la política de juventud.

Lo paradójico en todo esto, es que la solución que se vislumbra desde la esfera política, tiene que ver con la desaparición de dicho organismo, en vez de aportarle las capacidades necesarias para desarrollar sus funciones.

Volviendo a lo absurdo de la paradoja, es como si cada vez que se conocieran los resultados de las políticas educacionales, como solución a su deficiencia se pusiera en entredicho la existencia del Ministerio de Educación, por dar un ejemplo.

De esta forma, abrir la reflexión partiendo del análisis crítico de nuestra propia experiencia y la del resto de América Latina, rescatando (o elaborando) los aprendizajes obtenidos de este cruce entre la experiencia social juvenil y lo político administrativo, parece ser una ruta interesante de explorar.

Entonces, y abriendo una discusión prospectiva, ¿qué necesita el actual modelo de política de juventud chileno para promover la integración social de las y los jóvenes más excluidos? ${ }^{1}$ En relación a esta pregunta, tres reflexiones.

i) La forma v/s el fondo. En la medida que el Instituto Nacional de la Juventud opta por especializarse en un rol particular de investigación y evaluación de la política de juventud (Rodríguez, 2000), el modelo propone por descarte un ordenamiento administrativo de roles y funciones de las instituciones involucradas en la implementación de la política. Sin embargo no deja claro el sistema mediante el cual se construirán los sentidos y contenidos de la política de juventud, dejándolo como corolario de la legitimidad del organismo de coordinación central.

Desafortunadamente, en la medida en que el rol de coordinación de política públicas no se encuentre sólidamente legitimado, difícilmente será posible desde ahí generar una plataforma capaz de convocar a los diversos actores involucrados. Lo que aparece entonces es el desafío de construcción colectiva de sentidos, contenidos y orienta-

1 Como marco referencial se utiliza la caracterización de modelos institucionales de juventud elaborado por Ernesto Rodríguez y publicado en Última Década $\mathrm{N}^{\mathrm{0}} 13$ de septiembre del 2000. 
ciones de la política, la cual se complejiza y enriquece producto de la diversidad de actores involucrados en dicha gestión.

La base de este ejercicio debe sustentarse a partir del desarrollo de un pensamiento ético en relación al trabajo con jóvenes, el cual debe surgir desde las claves subjetivas de estructuración de los mundos juveniles para traducirse en una suma de señales que permitan dotar cualitativamente de pertinencia a la gestión pública en juventud como un todo coherente.

Sin embargo, este ejercicio de complementariedad sólo es efectivo y operativo en la esfera local, en tanto que es el mundo de la experiencia cotidiana en donde se produce el encuentro con sujetos reales, incluso cuando hacemos referencia a lo institucional.

Es en la localidad donde se corporaliza la institucionalidad y en donde se construyen las relaciones sociales, por tanto es ahí donde se requiere ejercitar la complementariedad y sumar energías. En definitiva se trata de generar instituciones a «escala humana».

ii) El descubrimiento del otro. Plantear que el nivel central asume el rol de coordinación de la política de juventud, implica reconocer y validar que otros actores tanto de la esfera pública como privada, asumen los roles de generación, construcción, implementación y evaluación de la política pública en los distintos niveles.

Durante quizás los últimos dos años, se han genera avances en la reflexión en torno a los elementos constitutivos de una política de juventud a nivel de presupuestos. De esta forma, para que la política cumpla su objetivo de integración social juvenil, debe al menos ser positiva en su visión de sujeto joven, propositiva en la medida que transfiere la decisión en torno a las estrategias de desarrollo juvenil a los propios jóvenes y local, pues es sólo en ese espacio territorial donde se ejerce ciudadanía (Silva, 1999).

Por lo tanto, el diseño e intervención de la política de juventud aparece en primera instancia como responsabilidad de actores locales, constituyéndose el municipio en un actor relevante para impulsar la generación de planes y políticas integrales de juventud y darle pertinencia y sustentabilidad necesaria.

Esta afirmación permite relevar el rol de los municipios en la generación de políticas sociales, y reviste sin duda una cambio cualitativo en la orientación de la política social en general.

Además, permite reconocer ciertas huellas, que dentro de un sistema de coordinación, sistematización y meta-análisis adecuado, permitiría desarrollar mejores estrategias para propiciar la integración 
social de las y los jóvenes, en la medida que permitiría aportar insumos para estudios longitudinales, por ejemplo.

iii) Políticas de juventud: el fantasma del sujeto. Principalmente, la política de juventud debe tener la capacidad de plantearse frente a sujetos reales. Esto no significa desconocer la construcción de sujeto elaborada por las ciencias sociales, sino que significa principalmente tener la capacidad de actualizar la relación tanto con el o la joven como con las diversas juventudes. Para lograr esto, es fundamental fortalecer la relación entre la sociedad civil joven y las instituciones en la localidad desde una óptica de respeto y promoción de derechos como base del ejercicio de ciudadanía.

De esta forma la política de juventud debe ser capaz de abrir canales de real participación juvenil como estrategia de legitimación y pertinencia de su propio accionar.

Haciendo una apuesta, con el objeto de articular el modelo de política de juventud es urgente desarrollar una estrategia que permita la co-construcción de sentidos de la política, no sólo para establecer roles y funciones diferenciadas en los distintos niveles, sino que principalmente para darle contenido a la política.

\section{MUNICIPIO Y POLÍTICAS LOCALES DE JUVENTUD}

En forma sencilla podemos entender que una política de juventud es aquella que surge producto de la materialización del esfuerzo de funcionarios y jóvenes, por entender la dimensión real y potencial de las realizaciones juveniles en el marco de su vida cotidiana; lo cual implica comprender cuáles son los factores asociados a ellas (que dinamizan y/u obstaculizan las experiencias juveniles), así como la postulación de pautas y guías para la acción tendientes a modificar dichos factores.

Valga el énfasis entonces, una política local de juventud no es la mera suma de programas expresados en el plan municipal, sino una propuesta estructurada de un conjunto de sentidos, orientaciones y acciones que desde su especificidad no sólo realizan su propósito particular, sino que contribuyen a fortalecer y legitimar nuevas experiencias de vida de los jóvenes, validando al sujeto joven en tanto actor social y destacando su participación como eje fundamental en las estrategias de integración social en la comuna.

Hecha esta declaración, cabe preguntarse qué funciones le competen a las Unidades Municipales de Juventud (UMJ en adelante) 
en el marco de articulación de una política social de juventud, desde el punto de vista prospectivo. A mi juicio ésas serían básicamente dos: i) Articular la política de juventud en la comuna; y, ii) Aportar insumos para generar un desarrollo local ascendente, esto fundamentalmente en el esfuerzo de construir desarrollo social desde l@s sujetos.

Para lograr orientar cualitativamente el rol del municipio en juventud en estos sentidos, es fundamental que la UMJ:

- Construya un marco de referencia y comprensión del mundo juvenil desde su cotidianeidad, factor de relevancia para la comprensión de diversidad y heterogeneidad del mundo juvenil.

- Desarrolle una estrategia de legitimación (Iglesis, 2000). Esto implica validar el trabajo de la UMJ en al menos tres niveles: con los jóvenes, al interior del municipio y con la institucionalidad pública y privada (esto último con el objeto de fortalecer un abordaje integral).

- Desarrolle un esfuerzo por generar políticas locales de juventud que impliquen la generación de planes de desarrollo juvenil desde l@s jóvenes y por tanto acotados a la realidad local.

- Finalmente, para lograr lo anterior, es indispensable promover la profesionalización de los equipos de juventud municipal, indispensable para fortalecer un abordaje integral centrado en la investigación-acción.

\section{EL PLAN DE DESARROLLO JUVENIL}

En relación a la elaboración de planes de desarrollo juvenil, y a partir de la experiencia acumulada en la comuna de Concepción a partir de 1998, creemos de importancia resguardar al menos los siguientes elementos:

- Un plan de desarrollo juvenil (PLADEJU en adelante) debe construirse a partir de la experiencia subjetiva de ciudadanía de las y los jóvenes.

- Debe considerar la estructuración de sentidos de las diversas juventudes, teniendo como partida la territorialidad, la micro-localidad. 
- Debe ser capaz de desarrollar la mejor estrategia de legitimación del sujeto joven a partir de la instalación de espacios de negociación directa entre los jóvenes y las autoridades institucionales. De esta forma resulta fundamental reconocer el conflicto como un elemento central de la dinámica social.

- Debe contar con respaldo social juvenil, vale decir, debe ser formulado, ejecutado y evaluado por 1@s mism@s jóvenes.

- Debe involucrar de manera dinámica lo económico, lo político, lo social y lo organizativo de las y los jóvenes.

- Debe contemplar la heterogeneidad juvenil, considerando diferencias de clase, género, territorialidad, etnia y otros factores relevantes en la construcción identidad de los jóvenes del 2000.

- Debe poseer un carácter participativo en cada nivel de planificación.

- Un PladeJU debe establecer claramente los ejes de intervención específicos para cada localidad o microlocalidad.

- Debe promover la generación de canales de comunicación específicos entre jóvenes e instituciones con el objeto de definir de manera adecuada la priorización de recursos.

- $\quad$ Debe ser capaz de concertar entre los diversos actores locales, el esquema de prioridades con el que se trabajará mientras esté vigente el plan, al igual que un esquema de responsabilidades entre los diversos actores públicos y privados involucrados.

- Debe ser capaz de identificar las alternativas de desarrollo del plan: esto es reconocer y estimar cuáles pueden ser los recursos reales y potenciales, y prever qué tipo de consecuencias institucionales, culturales y sociales puede provocar.

- La evaluación del PLADEJU debe ser vista como una re-lectura de la realidad y del proceso mismo, más que la comparación mecánica entre objetivos, metas y resultados; y debe tener como horizonte la instalación de procesos de integración social juvenil. 
De esta forma un plan de desarrollo juvenil no debe concebirse como una herramienta de planificación en el sentido tecnocrático, sino como una intervención social de amplio espectro, fundada en la investigación-acción y que tiene como objetivo estratégico generar plataformas de participación y de ejercicio ciudadano juvenil en la localidad.

Lo esencial en la generación e implementación del PLADEJU está fundado en la capacidad de éste de intervenir simultáneamente en la esfera social juvenil, comunitaria y político institucional de la comuna, generando espacios de diálogo, interlocución y negociación directa entre 1@s diversos actores. Su énfasis deberá estar puesto en la promoción y ampliación de derechos en 1@s jóvenes a través de la participación en un contexto de desarrollo local ascendente.

Diagrama de generación plan de desarrollo juvenil

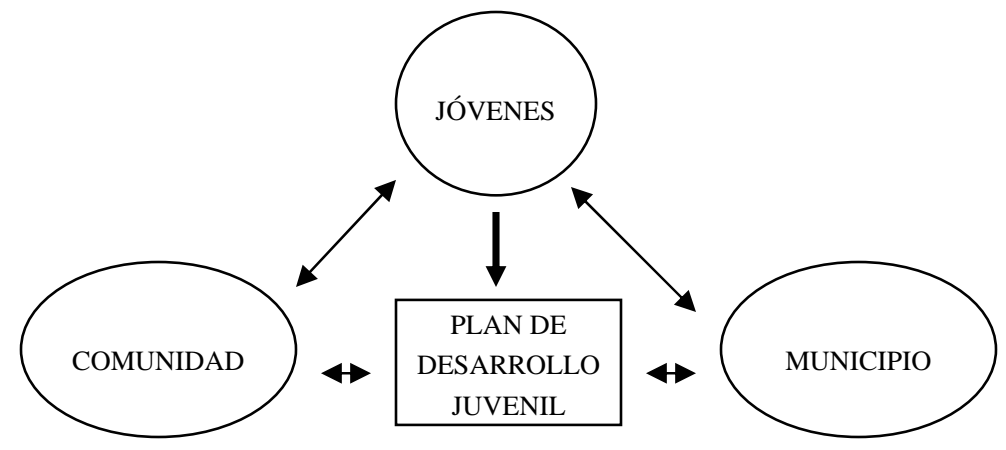

Indicadores de logro de la fase de diseño de un PLADEJU, lo constituyen:

i) Una amplia base social juvenil de apoyo al PLADEJU (la que debe ser capaz de trascender a núcleos de jóvenes que tienden a acercarse a las UMJ por razones de afinidad política con el/la alcalde de turno). Paralelamente es posible observar una proliferación y densificación de las redes juveniles de la comuna.

ii) Incorporación del PLADEJU como elemento prioritario de la agenda municipal. Esto significa principalmente que el PLADEJU tenga la capacidad de trascender a la UMJ e instalarse transversalmente en el municipio para el logro de sus metas. 
Es importante destacar que el PLADEJU es una herramienta de intervención social de mediano y largo plazo, en donde el énfasis está puesto en el proceso de construcción, generación y/o actualización de ciudadanía juvenil desde su cotidianeidad. De esta forma, el éxito del PLADEJU en cada una de sus etapas, no sólo está relacionado con el cumplimiento de las acciones planificadas, sino con la capacidad de 1@s propi@s jóvenes de activar circuitos de participación social legítimos y pertinentes a su realidad y a sus apuestas de construcción social.

Además, como toda investigación-acción participativa, el PLADEJU constituye una fuente generadora de aprendizajes para tod@s 1@s actoresinvolucrad@s.

\section{NOTAS PARA LA GENERACIÓN DE POLÍTICAS LOCALES DE JUVENTUD DESDE L@S JÓVENES: ALGUNAS PISTAS PARA CONSTRUIR RUTAS DESDE LO LOCAL}

i) La política local de juventud debe estructurarse a partir de la experiencia cotidiana de las y los jóvenes, posibilitando el tránsito entre lo privado-juvenil a lo público-comunal y de lo público-comunal a lo privado-juvenil, a través de la generación de plataformas de participación juvenil, que constituyan rutas de interlocución, negociación y actualización de la experiencia de ciudadanía en las y los jóvenes.

ii) De esta forma el diseño e implementación participativo de un plan de desarrollo juvenil permite orientar la política de juventud en la localidad, impactando simultáneamente en lo institucional y en la comunidad, construyendo un nuevo marco de relación.

iii) La generación de «PLADEJUS» aporta además un mapa claro que permite analizar nudos institucionales intra-comunales, aportando insumos para hacer lecturas a partir de la realidad comunal y regional.

iv) Esta experiencia replicada en otras comunas de diferentes regiones del país, aportaría un aprendizaje importantísimo en la construcción y elaboración del «contenido» de la política social de juventud, entendido como un conjunto de premisas básicas orientadas a trabajar desde la diversidad juvenil o desde las diversas juventudes del país. 
Modelo de política local de juventud

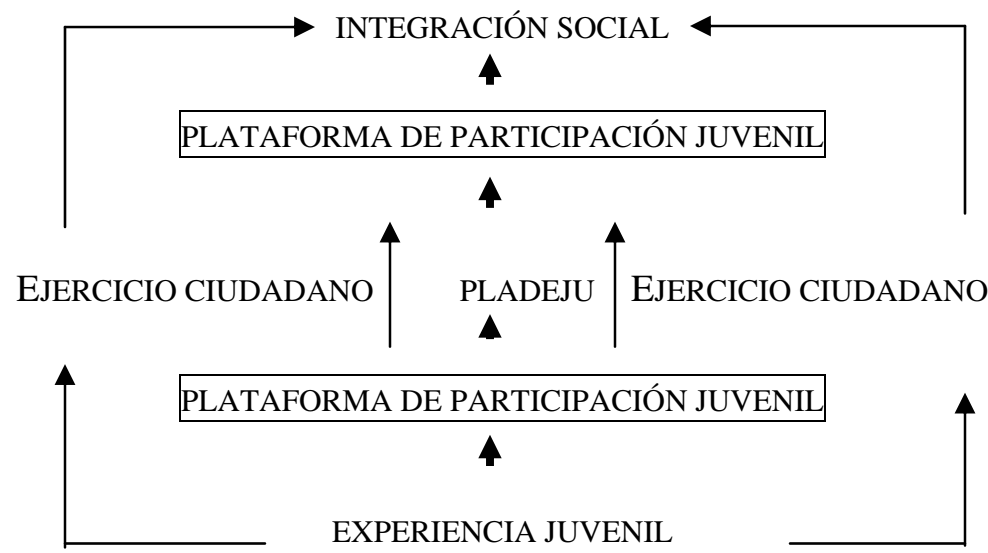

CONCEPCIÓN, FEBRERO DEL 2001

\section{REFERENCIAS BIBLIOGRÁFICAS}

Coljuventud (1993): Confabulando presentes. Santafé de Bogotá: Promover.

GONZÁLEZ, RAÚL (2000): «Análisis de la política social dirigida a los jóvenes». Santiago: Interjoven.

IGLESIS, ANDREA (2000): «Políticas locales de juventud: una mirada al fondo del ojo». Última Década $\mathrm{N}^{\circ} 12$. Viña del Mar: Ediciones CIDPA.

INTERJOVEN (2000): La Juventud en el triángulo de las Bermudas. Santiago: Interjoven.

RODRÍGUEZ, ERNESTO (2000): «Juventud, participación y desarrollo en América Latina: desafíos y prioridades en el comienzo de un nuevo siglo». Notas presentadas en el II Foro Juvenil del Mercosur, Bolivia y Chile sobre Participación Juvenil.

SilVA, ClAUdiO (2000): «Reflexiones y aportes a una política comunal —a escala humana- en juventud». Última Década №12. Viña del Mar: Ediciones CIDPA. 